\title{
Predictors of cognitive decline in Alzheimer's disease and mild cognitive impairment using the CAMCOG: a five-year follow-up
}

\author{
Josep L. Conde-Sala, ${ }^{1}$ Josep Garre-Olmo, 2,3 Joan Vilalta-Franch, 3,4 \\ Jordi Llinàs-Reglà, ${ }^{4}$ Oriol Turró-Garriga, ${ }^{3,4}$ Manuela Lozano-Gallego, ${ }^{4}$ \\ Marta Hernández-Ferrándiz, ${ }^{4}$ Immaculada Pericot-Nierga ${ }^{4}$ and \\ Secundino López-Pousa ${ }^{3,4}$ \\ ${ }^{1}$ Faculty of Psychology, University of Barcelona, Barcelona, Spain \\ ${ }^{2}$ Department of Psychology, University of Girona, Girona, Spain \\ ${ }^{3}$ Research Unit, Institut d'Assistència Sanitària, Salt, Spain \\ ${ }^{4}$ Dementia Unit, Hospital de Santa Caterina, Institut d'Assistència Sanitària, Salt, Spain
}

ABSTRACT

Background: There are discrepant findings regarding which subscales of the Cambridge Cognitive Examination (CAMCOG) are able to predict cognitive decline. The study aimed to identify the baseline CAMCOG subscales that can discriminate between patients and predict cognitive decline in Alzheimer's disease (AD) and mild cognitive impairment (MCI).

Methods: This was a five-year case-control study of patients with cognitive impairment and a control group. Participants were grouped into AD $(n=121)$, MCI converted to dementia (MCI-Ad, $n=43)$, MCI-stable (MCI-St, $n=66$ ), and controls (CTR, $n=112$ ). Differences in the mean scores obtained by the four groups were examined. Receiver operating characteristic curves were used to compare subscale scores in the $\mathrm{AD}$ and MCI-Ad groups with those of controls. The influence of age, gender, schooling, and depression on baseline subscale scores was assessed.

Results: Of the CAMCOG subscales, Orientation and Memory (learning and recent) (OR + MEM) showed the highest discriminant capacity in the baseline analysis of the four groups. This baseline analysis indicated that $\mathrm{OR}+\mathrm{MEM}$ was the best predictor of conversion to $\mathrm{AD}$ in the MCI-Ad group (area under the curve, AUC $=0.81$ ), whereas the predictive capacity of the global MMSE and CAMCOG scores was poor (AUC = 0.59 and 0.53 , respectively).

Conclusions: In the baseline analysis, the Orientation and Memory (learning and recent) subscales showed the highest discriminant and predictive capacity as regards both cognitive decline in the AD group and conversion to $\mathrm{AD}$ among MCI-Ad patients. This was not affected by age, gender, schooling, or depression.

Key words: risk factors, dementia, neuropsychological tests, conversion to dementia, orientation, memory, aging

\section{Introduction}

The identification of biological and neuropsychological markers that can predict cognitive decline at the preclinical stage is an important area of research. Studies conducted with the Cambridge Cognitive Examination (CAMCOG) have reported variable results as regards which subscales might discriminate between diagnostic categories and predict cognitive decline.

Correspondence should be addressed to: Josep Lluís Conde-Sala, University of Barcelona, Passeig Vall d'Hebron, 171, 08035 Barcelona, Spain. Phone: +3493-312-58-14; Fax: +34-93-402-13-68. Email: jllconde@ub.edu. Received 15 Jun 2011; revision requested 27 Jul 2011; revised version received 7 Sep 2011; accepted 25 Sep 2011. First published online 26 January 2012.
Global CAMCOG scores obtained the best diagnostic accuracy in differentiating between normal patients, those with mild cognitive impairment (MCI), and patients with Alzheimer's disease (AD) (Heinik and Shaikewitz, 2009), and were better at discriminating between $\mathrm{AD}$ and MCI patients than between MCI and normal individuals (Nunes et al., 2008). The subscales that best discriminated between dementia and non-dementia were Memory, Orientation, and Attention/Calculation, whereas Abstraction, Language, and Perception presented the highest discrepancies among studies (Lozano-Gallego et al., 1999; Nielsen et al., 1999; Schmand et al., 2000; Williams et al., 2003). 
In the conversion from normal aging to MCI, one longitudinal study reported a greater decline associated with older age and the subscales Language (expression) and Memory (learning), which were predictors of the conversion time (Oulhaj et al., 2009). As regards predicting the conversion from $\mathrm{MCI}$ to $\mathrm{AD}$, there is a broader consensus over the predictive value of the Orientation and Memory subscales and the global CAMCOG score at baseline, but greater disagreement in relation to the Language, Perception, Abstraction, and Attention/Calculation subscales (Cabranes et al., 2004; Marcos et al., 2006; Armas, 2009; Gallagher et al., 2010). These discrepancies could be due to the influence of socio-demographic factors such as age or education, if the samples studied were heterogeneous with respect to these variables. At all events, it would clearly be helpful to clarify that subscales are indeed able to identify at baseline those patients who are subsequently most likely to suffer cognitive decline, as well as those subscales that show the greatest decline from a longitudinal perspective.

The main hypothesis of the present research was that it would be possible, at baseline, to identify differential elements in the CAMCOG subscales that could be used to predict cognitive decline, especially in subjects with MCI. This is relevant since the overall score has been shown to be a poor discriminator. Consequently, the study objectives were to identify, in a sample of $\mathrm{AD}, \mathrm{MCI}$, and normal aging subjects who were homogeneous in terms of socio-demographic variables: (1) the CAMCOG subscales with the highest discriminating capacity at baseline in terms of predicting cognitive decline in $\mathrm{AD}$ and MCI; (2) the effect of age, schooling, gender, and depression on baseline subscale scores; and (3) the subscales that show the greatest decline over a five-year period in $\mathrm{AD}$ and $\mathrm{MCI}$.

\section{Methods}

\section{Design and sample population}

A case-control longitudinal study was carried out with patients who had been seen as outpatients at the Memory and Dementia Assessment Unit (UVAMID) of the Santa Caterina Hospital in Girona, Spain and who had undergone neuropsychological assessment over five consecutive years.

Patients were diagnosed on the basis of standard clinical criteria for each group ( $\mathrm{AD}$ and $\mathrm{MCI}$ ), independently of their CAMCOG results. They were then classified, according to their final diagnosis, as patients with $\mathrm{AD}$, patients with MCI converted to $\mathrm{AD}$ (MCI-Ad), and patients with stable MCI (MCI-St). Those with AD met DSM-
IV-TR criteria (American Psychiatric Association, 2001), while probable AD patients were classified according to the NINCDS-ADRDA criteria (McKhann et al., 1984). Patients with MCI met the European AD Consortium (EADC) criteria for MCI (Portet et al., 2006):

1. cognitive complaints coming from the patients or their families;

2. the reporting of a decline in cognitive functioning relative to previous abilities during the past year by the patient or informant;

3. cognitive disorders as evidenced by clinical evaluation (impairment in memory or in another cognitive domain);

4. absence of major repercussions on daily life (the patient may, however, report difficulties concerning complex day-to-day activities);

5. absence of dementia.

The control group (CTR) was drawn from an epidemiological field study on the prevalence of dementias in a rural area of the province of Girona, Spain (Vilalta-Franch et al., 2000; LópezPousa et al., 2004). There were initially 141 CTR individuals without dementia, assessed over a period of five years. Of these, only those cases with complete neuropsychological test results were selected, resulting in a group of 112 individuals.

Patient data corresponded to the period between 1995 and 2009. The CTR data were obtained between 1990 and 1995 . The final sample therefore consisted of four groups: AD $(n=121)$, MCI-Ad $(n=43)$, MCI-St $(n=66)$, and CTR $(n=112)$.

\section{Measurements}

SOCIO-DEMOGRAPHIC DATA

Data on the patients' age, gender, marital status, and education were gathered in the hospital unit. For controls, data were derived from the Cambridge Mental Disorders of the Elderly Examination (CAMDEX; Roth et al., 1986; Vilalta-Franch et al., 1990) that had been applied to all subjects in the epidemiological field study.

\section{INSTRUMENTS}

The neuropsychological examination of patients and controls included the following tests:

1. Mini-Mental State Examination (MMSE). This is a brief cognitive test whose score ranges between 0 and 30, the cut-off for cognitive impairment being 21/22 (Folstein et al., 1975).

2. CAMCOG. This evaluates several cognitive functions and its total score ranges between 0 and 105-107 (the latter depending on whether the original or revised version is used). The lower the score the greater the cognitive impairment. The cutoff score for cognitive impairment in the Spanish 
population is 69/70 (Vilalta-Franch et al., 1990). The CAMCOG subscales are Orientation, Memory (learning, recent, and remote), Language (comprehension and expression), Attention/Calculation, Praxis, Perception, and Abstraction. The original version of the CAMCOG was applied to the control group (Roth et al., 1986; Vilalta-Franch et al., 1990), whereas the revised version was administered to patients (Roth et al., 1998; Lozano-Gallego et al., 2000). When computing scores, one item from the Perception subscale (tactile recognition of coins) was eliminated from the original version as it does not form part of the revised CAMCOG.

3. Blessed Dementia Rating Scale (BDRS; Blessed et al., 1968). Scores on the first two subscales of this instrument were used to evaluate deficits in activities of daily living (ADL) (A) and habits (B). The maximum deficit score for these subscales combined is $17(\mathrm{~A}=8 ; \mathrm{B}=9)$. The cut-off value for the Spanish population is 1.5 for the two subscales combined (A + B; Peña-Casanova et al., 2005).

4. CAMDEX-R Depression Scale. This has a maximum score of 27 and a cut-off value of $7 / 8$ for the Spanish population (Vilalta-Franch et al., 1990).

\section{Procedure}

The research team was formed by clinicians with both neuropsychology training and clinical experience. The neuropsychological assessment of patients (AD, MCI-Ad, and MCI-St) was performed by the team of neuropsychologists from the hospital unit. In addition to diagnosis, a second inclusion criterion was having been assessed over a consecutive five-year period. In the epidemiological field study, the neuropsychological assessment of the control group was conducted in each participant's home.

During the five-year period, individuals with dementia (AD and MCI-St) underwent an average of 4.8 assessments (range $2-6$ ), while those without dementia (MCI-St and CTR) had an average of 3.1 assessments (range $2-6$ ).

\section{Statistical analysis}

An analysis was conducted of the CAMCOG subscale scores obtained by the four groups. Differences in mean scores were compared using the Kruskal-Wallis $\left(\chi^{2}\right)$, Mann-Whitney U $(z)$, and Wilcoxon $(z)$ tests because none of the subscales fulfilled the criteria for normality (KolmogorovSmirnov and Shapiro-Wilks tests). In the multiple comparisons, Bonferroni correction was applied to the $p$ values. The effect size (Cohen's $d$ ) was calculated whenever any differences were significant.

An analysis of the receiver operating characteristic (ROC) curves was also conducted to identify the subscales with the greatest predictive capacity, determined by comparing the baseline and final scores of the $\mathrm{AD}$ versus CTR groups and the MCIAd versus CTR groups.

Finally, a stepwise multivariate linear regression analysis was carried out, introducing as dependent variables the baseline scores on each subscale and as independent variables the subjects' age, gender, years of schooling, and depression. The aim here was to identify the subscales most affected by these variables. Results were considered statistically significant at a $p$ value of 0.05 .

\section{Results}

\section{Socio-demographic data}

The mean age of the sample at baseline was 74.3 years $(\mathrm{SD}=6.2)$, with men accounting for $31.3 \%$ $(n=107)$ and women $68.7 \%(n=235)$. As regards education, $20.8 \%(n=71)$ of the sample were either illiterate or had no formal schooling, $18.4 \%(n=$ 63) had received one to five years of education, $50.0 \%(n=171)$ had attended school for six to eight years, and $10.8 \%(n=37)$ had received more than eight years of education. There were no significant differences between the four groups in terms of age $\left(\chi^{2}=1.7 ; p=0.628\right)$, gender $\left(\chi^{2}=1.6 ; p=0.649\right)$, marital status $\left(\chi^{2}=0.9 ; p=0.810\right)$, or schooling $\left(\chi^{2}=2.6 ; p=0.441\right)$.

\section{Clinical data}

ADL/HABITS

The mean scores at baseline on the two subscales $(\mathrm{A}+\mathrm{B})$ of the BDRS were as follows: $\mathrm{AD}=2.27$ (1.4), MCI-Ad $=1.37$ (1.0), MCI-St $=1.24$ (1.0), and CTR $=0.34(0.6)$. The corresponding mean scores at final assessment were $\mathrm{AD}=5.95(2.6)$, MCI-Ad $=4.18$ (1.7), MCI-St $=2.46(1.4)$, and CTR $=0.99(1.1)$.

\section{SUBTyPES OF MCI}

At baseline, the amnesic subtype (single and multiple domains) accounted for $90.7 \%$ of the MCI-Ad group and $80.3 \%$ of the MCI-St group, while the non-amnesic subtype (single and multiple domains) accounted for $9.3 \%$ of the MCI-Ad group and $19.7 \%$ of the MCI-St group. These subtypes were classified by taking into account, for each subscale, the deficit scores that were more than one standard deviation greater than the scores obtained by controls.

\section{DEPRESSION}

Application of the CAMDEX-R Depression Scale revealed the following percentages of depressed 
subjects in each group: $\mathrm{AD}=33.1 \%, \mathrm{MCI}-\mathrm{Ad}=$ $44.2 \%$, MCI-St $=57.6 \%$, and CTR $=23.2 \%$. However, the presence or absence of depression was not significantly related, in any of the groups, with baseline or final scores on the CAMCOG, or with the overall rate of cognitive decline.

\section{Scores on the CAMCOG subscales}

Table 1 shows the mean scores on the CAMCOG subscales at baseline and after five years. When presenting these results in Figure 1, $T(10 z+50)$ scores are used so as to reflect better the relative differences between the four study groups.

At baseline, the $\mathrm{AD}$ group scored lower than the other three groups on all the subscales. The MCIAd group scored significantly higher than the MCISt group on Language (expression) $(z=-3.4 ; p<$ $0.001)$, Attention/Calculation $(z=-2.5 ; p=$ $0.011)$, and Abstraction $(z=-2.1 ; p=0.035)$ and higher than the CTR group on Praxis $(z=$ $-3.0 ; p=0.002)$. These higher comparative scores in the MCI-Ad group produce a confounding effect in the baseline assessment. The only variable on which the MCI-Ad group scored lower than the groups without dementia was Memory (learning), this being the case with respect to both the MCI-St group $(z=2.2 ; p=0.025)$ and the CTR group $(z=4.0 ; p<0.001)$. The MCI-St group scored lower than the CTR group on Orientation $(z=$ 6.5 ; $p<0.001)$, Memory (recent) $(z=3.5$; $p<$ $0.001)$, and Language (expression) $(z=2.0 ; p=$ $0.038)$, but higher on Praxis $(z=-2.1 ; p=$ 0.035).

The only subscales on which the baseline scores increased progressively from $\mathrm{AD}$ to MCI-Ad to MCI-St to CTR were Orientation and Memory (learning and recent). By grouping these subscales into a new variable, OR + MEM, it was possible to achieve a greater discrimination between the four groups: AD versus MCI-Ad (16.1 [5.9] vs. 20.4 [4.2]; $z=4.0, p=0.001 ; d=0.84)$; MCI-Ad versus MCI-St (20.4 [4.2] vs. 22.3 [3.6]; $z=2.1$, $p=0.033 ; d=0.48)$; and MCI-St versus CTR (22.3 [3.6] vs. 24.6 [3.0]; $z=4.1, p<0.001 ; d=$ 0.69). A notable difference was also observed when comparing the baseline OR + MEM scores obtained by the MCI-Ad group (the most difficult to diagnose) with those of controls (20.4 [4.2] vs. 24.6 [3.0]; $z=5.5, p<0.001 ; d=1.15)$.

The difficulty in identifying differential scores at baseline decreased after five years. By the time of the final assessment, the groups tended to cluster into dementia (AD and MCI-Ad) and nondementia (MCI-St and CTR), and all the subscales presented significant differences between these groups with and without dementia. The greatest difference continued to be on the combined OR + MEM variable: dementia versus non-dementia (10.2 [4.9] vs. 23.6 [3.8]; $z=15.1, p<0.001$; $d=3.05)$. Between the $\mathrm{AD}$ and MCI-Ad groups, there were no significant differences on any of the subscales, including OR + MEM: AD versus MCIAd (9.9 [4.8] vs. 10.9 [5.1]; $z=1.0, p=0.305)$. Comparison of the MCI-St and CTR groups revealed that the significant differences found on $\mathrm{OR}+\mathrm{MEM}$ at baseline were maintained after five years: MCI-St versus CTR (21.7 [4.2] vs. 24.7 $[3.1] ; z=4.7, p<0.001 ; d=0.81)$.

\section{ROC curves}

ROC curves were used to carry out a predictive analysis of the subscales, comparing both the $\mathrm{AD}$ and MCI-Ad groups with the CTR group (Figure 2 and Table S1, the latter published online as supplementary material attached to the electronic version of this paper at http://www.journals.cambridge.org/ipg).

At baseline, the $\mathrm{AD}$ group had an area under the curve (AUC) above 0.70 on the subscales Orientation (AUC $=0.86$ ), Memory (learning) $(\mathrm{AUC}=0.84)$, and Memory (recent) $(\mathrm{AUC}=$ 0.77), while the highest AUC value corresponded to the combined variable OR + MEM $(\mathrm{AUC}=0.89)$. Global scores on the MMSE (AUC $=0.80)$ and the CAMCOG (AUC $=0.74$ ) showed an acceptable predictive capacity. In the final assessment, these subscales showed an excellent predictive capacity, with AUC values equal to or higher than 0.94 .

Baseline results for the MCI-Ad group showed that the same subscales again had a predictive capacity, the AUCs being acceptable although slightly lower than those of the $\mathrm{AD}$ group: Orientation, AUC $=0.79$; Memory (learning), $\mathrm{AUC}=0.73$; Memory (recent), $\mathrm{AUC}=0.74$; and $\mathrm{OR}+\mathrm{MEM}, \mathrm{AUC}=0.81$. Global scores on the MMSE (AUC $=0.59$ ) and CAMCOG (AUC $=$ 0.53 ) had no predictive capacity in the MCI-Ad group. In the final assessment, these subscales had an excellent predictive capacity, with AUC values above 0.94 , the same as in the $\mathrm{AD}$ group.

\section{Longitudinal improvement and decline}

Figure 3 and Table S2 (the latter published online as supplementary material attached to the electronic version of this paper at http://www.journals.cambridge.org/ipg) show the improvement or decline produced between baseline and final scores, with an interval of five years. The AD and MCI-Ad groups showed a significant decline on all the subscales, most notably ( $>30 \%)$ on Orientation, Memory (learning, recent, and remote), and Attention/Calculation; moderately 
Table 1. CAMCOG subscale scores at baseline and after five years

\begin{tabular}{|c|c|c|c|c|c|c|c|c|c|c|c|}
\hline & \multirow{3}{*}{$\begin{array}{l}\text { TOP } \\
\text { SCORE }\end{array}$} & \multicolumn{4}{|c|}{ MEAN（SD） } & \multicolumn{6}{|c|}{ INTERGROUPS DIFFERENCES } \\
\hline & & \multirow{2}{*}{$\begin{array}{l}\text { 1. AD } \\
(N=121)\end{array}$} & \multirow{2}{*}{$\begin{array}{l}\text { 2. MCI-AD } \\
(N=43)\end{array}$} & \multirow{2}{*}{$\begin{array}{l}\text { 3. MCI-ST } \\
(N=66)\end{array}$} & \multirow{2}{*}{$\begin{array}{l}\text { 4. CTR } \\
(N=112)\end{array}$} & \multicolumn{2}{|c|}{1 vs. 2} & \multicolumn{2}{|c|}{2 vs. 3} & \multicolumn{2}{|c|}{3 vs. 4} \\
\hline & & & & & & $P$ & $D$ & $P$ & $D$ & $P$ & $D$ \\
\hline \multicolumn{12}{|l|}{ Baseline } \\
\hline MMSE & 30 & $18.5(3.5)$ & $21.7(3.3)$ & $21.0(2.9)$ & $22.8(3.6)$ & $<0.001$ & 0.94 & 0.186 & & 0.001 & 0.55 \\
\hline CAMCOG & 105 & $60.3(11.2)$ & $71.2(8.6)$ & $68.1(7.8)$ & $71.6(12.2)$ & $<0.001^{*}$ & 1.09 & $0.858^{*}$ & & $0.215^{*}$ & \\
\hline Orientation & 10 & $7.0(2.0)$ & $8.1(1.4)$ & $8.4(1.2)$ & $9.5(0.9)$ & 0.001 & 0.63 & 0.311 & & $<0.001$ & 1.03 \\
\hline Learning memory & 17 & $6.8(3.7)$ & $9.6(2.8)$ & $10.8(2.7)$ & $11.7(2.3)$ & $<0.001$ & 0.85 & 0.025 & 0.43 & 0.078 & \\
\hline Recent memory & 4 & $2.2(1.2)$ & $2.7(1.0)$ & $2.9(0.8)$ & $3.4(0.7)$ & 0.031 & 0.45 & 0.144 & & $<0.001$ & 0.66 \\
\hline Remote memory & 6 & $2.8(1.5)$ & $3.5(1.2)$ & $3.1(1.4)$ & $3.5(1.4)$ & 0.011 & 0.51 & 0.195 & & 0.167 & \\
\hline Comprehension L & 9 & $7.3(1.3)$ & $7.9(0.9)$ & $7.6(1.0)$ & $7.8(1.2)$ & 0.030 & 0.53 & 0.303 & & 0.145 & \\
\hline Expression L & 21 & $13.5(2.5)$ & $15.2(2.4)$ & $13.5(2.0)$ & $14.3(2.5)$ & 0.001 & 0.69 & $<0.001$ & -0.77 & 0.038 & 0.35 \\
\hline Attention/Calculation & 9 & $3.6(2.2)$ & $5.3(2.5)$ & $4.1(2.2)$ & $4.7(2.5)$ & $<0.001$ & 0.72 & 0.011 & -0.51 & 0.204 & \\
\hline Praxis & 12 & $8.4(1.8)$ & $9.4(1.7)$ & $9.0(1.6)$ & $8.1(2.5)$ & 0.001 & 0.57 & 0.077 & & 0.035 & -0.42 \\
\hline Perception & 9 & $6.0(1.4)$ & $5.9(1.6)$ & $5.9(1.2)$ & $5.7(1.4)$ & 0.696 & & 0.376 & & 0.453 & \\
\hline Abstraction & 8 & $2.0(2.2)$ & $3.2(2.2)$ & $2.3(2.0)$ & $2.7(2.2)$ & 0.002 & 0.54 & 0.035 & -0.42 & 0.215 & \\
\hline $\mathrm{OR}+\mathrm{MEM}$ & 31 & $16.1(5.9)$ & $20.4(4.2)$ & $22.3(3.6)$ & $24.6(3.0)$ & $<0.001$ & 0.84 & 0.033 & 0.48 & $<0.001$ & 0.69 \\
\hline \multicolumn{12}{|c|}{ After a period of five years } \\
\hline MMSE & 30 & $14.7(3.6)$ & $16.4(4.0)$ & $21.3(2.7)$ & $22.8(3.4)$ & 0.016 & 0.44 & $<0.001$ & 1.43 & 0.006 & 0.48 \\
\hline CAMCOG & 105 & $45.8(11.5)$ & $48.4(12.3)$ & $68.1(9.2)$ & $72.2(11.3)$ & $1.000^{*}$ & & $<\mathbf{0 . 0 0 1}^{*}$ & 1.81 & $0.097^{*}$ & \\
\hline Orientation & 10 & $4.4(2.1)$ & $5.0(2.2)$ & $8.3(1.5)$ & $9.4(0.8)$ & 0.136 & & $<\mathbf{0 . 0 0 1}$ & 1.75 & $<0.001$ & 0.91 \\
\hline Learning memory & 17 & $4.6(2.8)$ & $4.8(2.7)$ & $10.7(2.6)$ & $11.9(2.4)$ & 0.548 & & $<0.001$ & 2.22 & 0.004 & 0.48 \\
\hline Recent memory & 4 & $0.8(0.9)$ & $1.0(1.1)$ & $2.6(1.0)$ & $3.3(0.8)$ & 0.736 & & $<0.001$ & 1.52 & $<0.001$ & 0.77 \\
\hline Remote memory & 6 & $1.8(1.2)$ & $2.0(1.1)$ & $2.9(1.3)$ & $3.5(1.3)$ & 0.247 & & 0.001 & 0.74 & 0.017 & 0.46 \\
\hline Comprehension L & 9 & $6.8(1.5)$ & $6.7(1.6)$ & $7.5(1.2)$ & $7.8(1.1)$ & 0.915 & & 0.011 & 0.56 & 0.113 & \\
\hline Expression L & 21 & $11.3(2.5)$ & $11.7(3.6)$ & $13.8(2.1)$ & $14.0(2.4)$ & 0.112 & & 0.003 & 0.71 & 0.772 & \\
\hline Attention/Calculation & 9 & $2.7(2.1)$ & $3.1(2.3)$ & $4.2(2.2)$ & $4.6(2.4)$ & 0.410 & & 0.008 & 0.48 & 0.508 & \\
\hline Praxis & 12 & $6.8(2.0)$ & $7.0(1.9)$ & $8.8(1.6)$ & $8.6(2.1)$ & 0.913 & & $<0.001$ & 1.02 & 0.649 & \\
\hline Perception & 9 & $4.5(1.5)$ & $4.6(1.8)$ & $5.8(1.3)$ & $5.6(1.5)$ & 0.593 & & $<0.001$ & 0.76 & 0.280 & \\
\hline Abstraction & 8 & $1.7(1.7)$ & $2.1(1.8)$ & $3.0(1.7)$ & $3.0(2.1)$ & 0.143 & & 0.010 & 0.51 & 0.962 & \\
\hline $\mathrm{OR}+\mathrm{MEM}$ & 31 & $9.9(4.8)$ & $10.9(5.1)$ & $21.7(4.2)$ & $24.7(3.1)$ & 0.305 & & $<0.001$ & 2.31 & $<0.001$ & 0.81 \\
\hline
\end{tabular}

$\mathrm{AD}=$ Alzheimer's disease; MCI-Ad $=$ mild cognitive impairment converted to $\mathrm{AD} ; \mathrm{MCI}-\mathrm{St}=$ mild cognitive impairment-stable; $\mathrm{CTR}=$ control group; $\mathrm{OR}+\mathrm{MEM}=\mathrm{Orientation}+\mathrm{Memor}$ (learning and recent); $\mathrm{L}=$ Language; $D=$ Cohen's $d$.

$\mathrm{P}=$ Mann-Whitney $\mathrm{U} ; p$ value adjusted with Bonferroni correction for multiple comparisons $\leq 0.017$. *ANOVA; $p$ value adjusted with Bonferroni correction for multiple comparisons. Significant $p$ values in bold. 

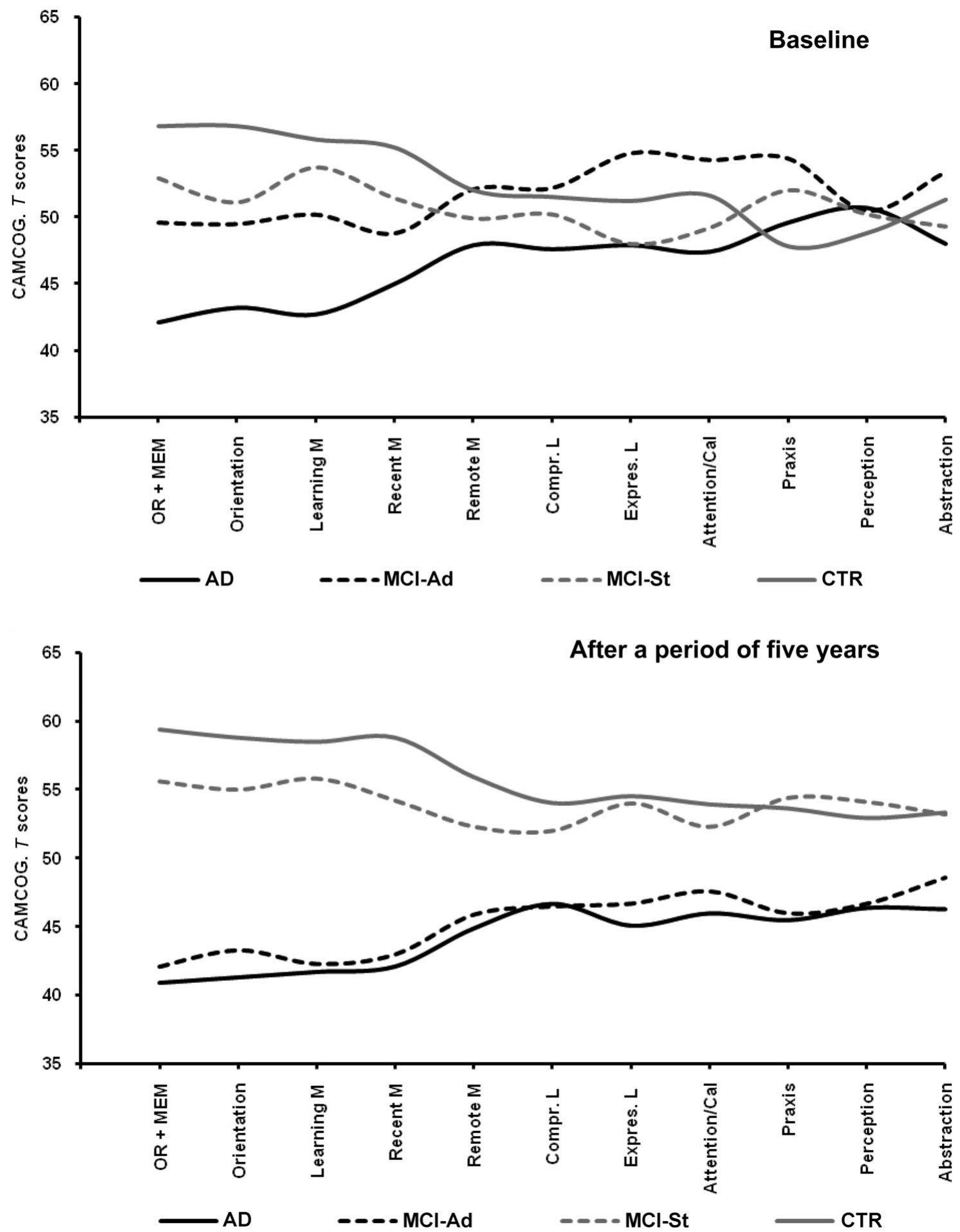

Figure 1. CAMCOG subscale $T$ scores at baseline and after five years. $A D=$ Alzheimer's disease; $M C I-A d=$ mild cognitive impairment converted to $\mathrm{AD} ; \mathrm{MCl}-\mathrm{St}=$ mild cognitive impairment-stable; $\mathrm{CTR}=$ control group; $\mathrm{OR}+\mathrm{MEM}=$ Orientation + Memory (learning and recent); $M=$ Memory; $L=$ Language; Compr. = comprehension; Expres. = expression; Cal. = calculation.

(20-25\%) on Perception, Language (expression), Praxis, and Abstraction; and mildly (10\%) on Language (comprehension).

In general, subscale scores in the MCI-St and CTR groups remained stable between the two assessment points. The MCI-St group showed a slight decline in recent memory (11.7\%) and an improvement on the Abstraction subscale (32.6\%), while the CTR group improved slightly on Praxis $(6.7 \%)$. 
$\operatorname{AD}(N=121) / C T R(N=112)$

Baseline

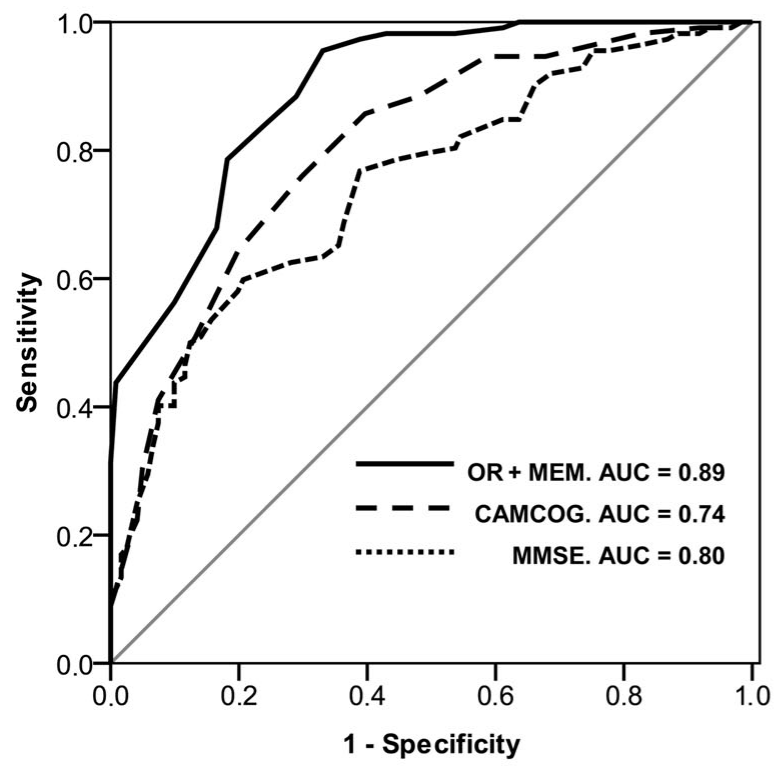

After a period of five years

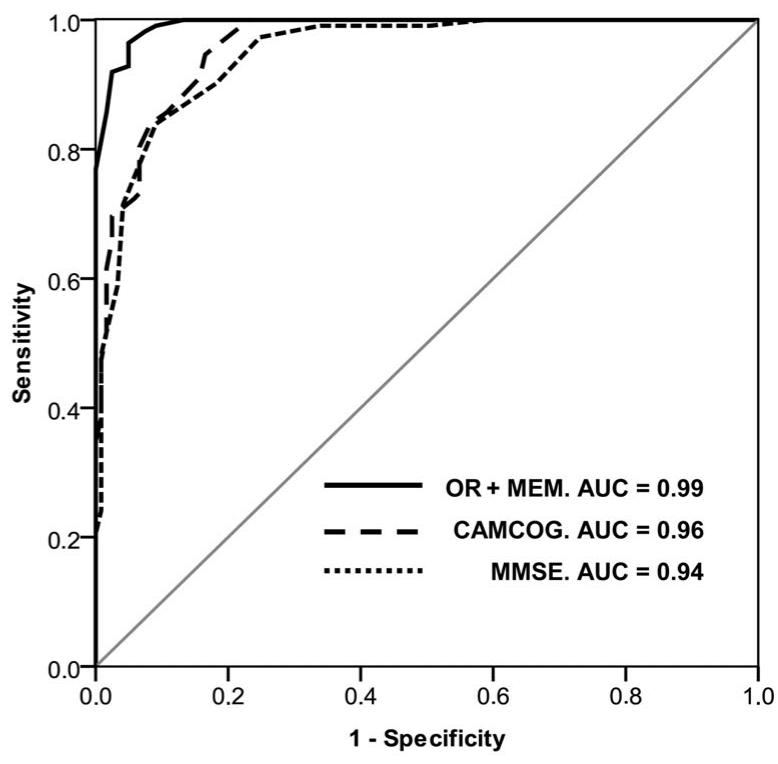

$\operatorname{MCI}-A d(N=43) / C T R(N=112)$

Baseline

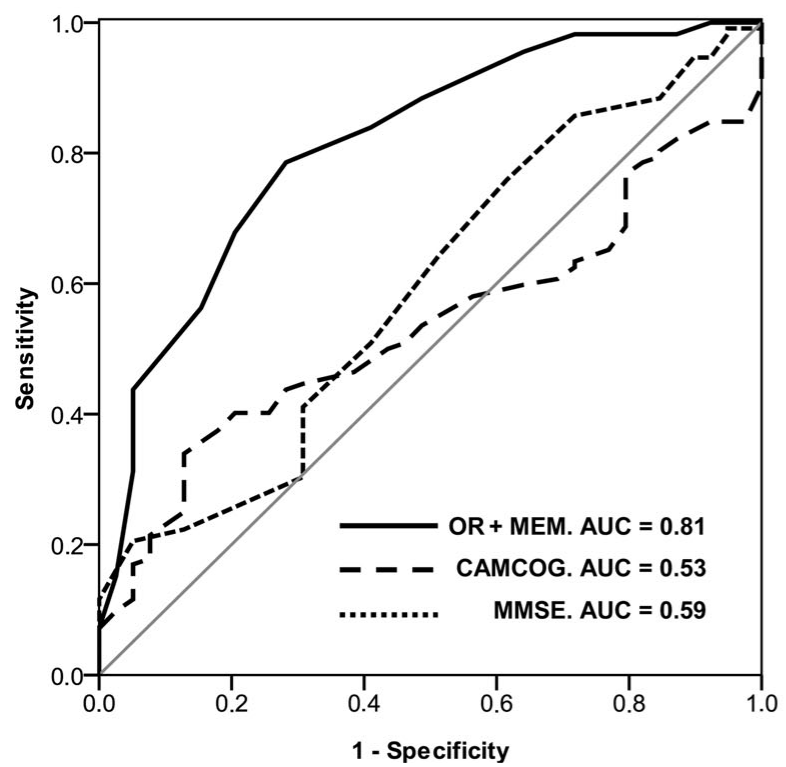

After a period of five years

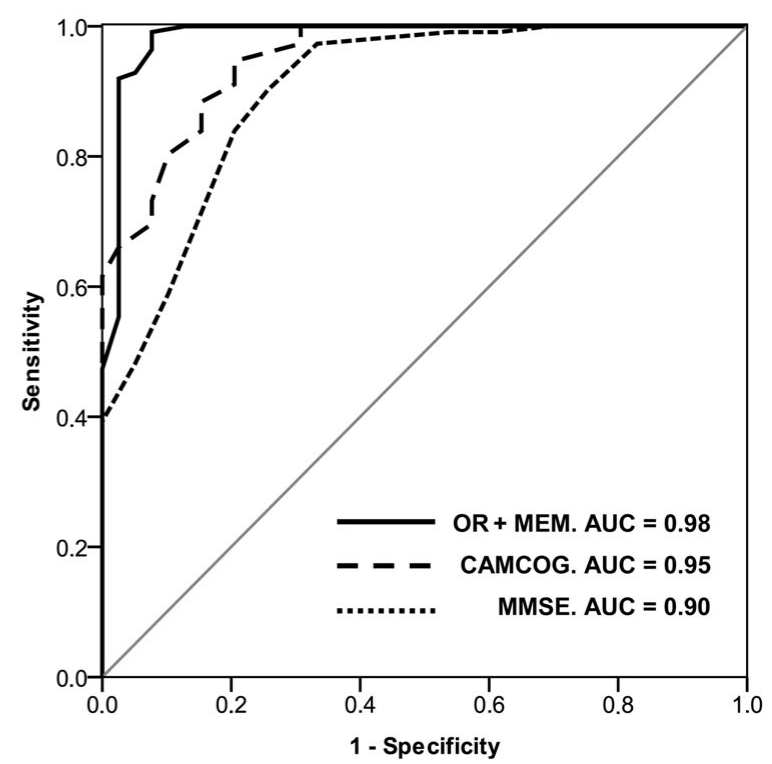

Figure 2. ROC curves. Ability to predict cognitive decline in the $A D$ and $M C I-A d$ groups in relation to the control group. AD = Alzheimer's disease; $M C I-A d=$ mild cognitive impairment converted to $A D ; C T R=$ control group; $O R+M E M=$ Orientation + Memory (learning and recent); $A U C=$ area under curve.

\section{Effect of age, education, gender, and} depression on baseline subscale scores

A multivariate linear regression analysis was performed in order to identify any subscales that were affected by these variables. The dependent variables were baseline scores on each of the subscales, while the independent variables were age, gender, years of schooling, and depression (see Table 2).

In the groups with dementia, neither the Orientation nor Memory (learning and recent) subscale nor the OR + MEM variable was 


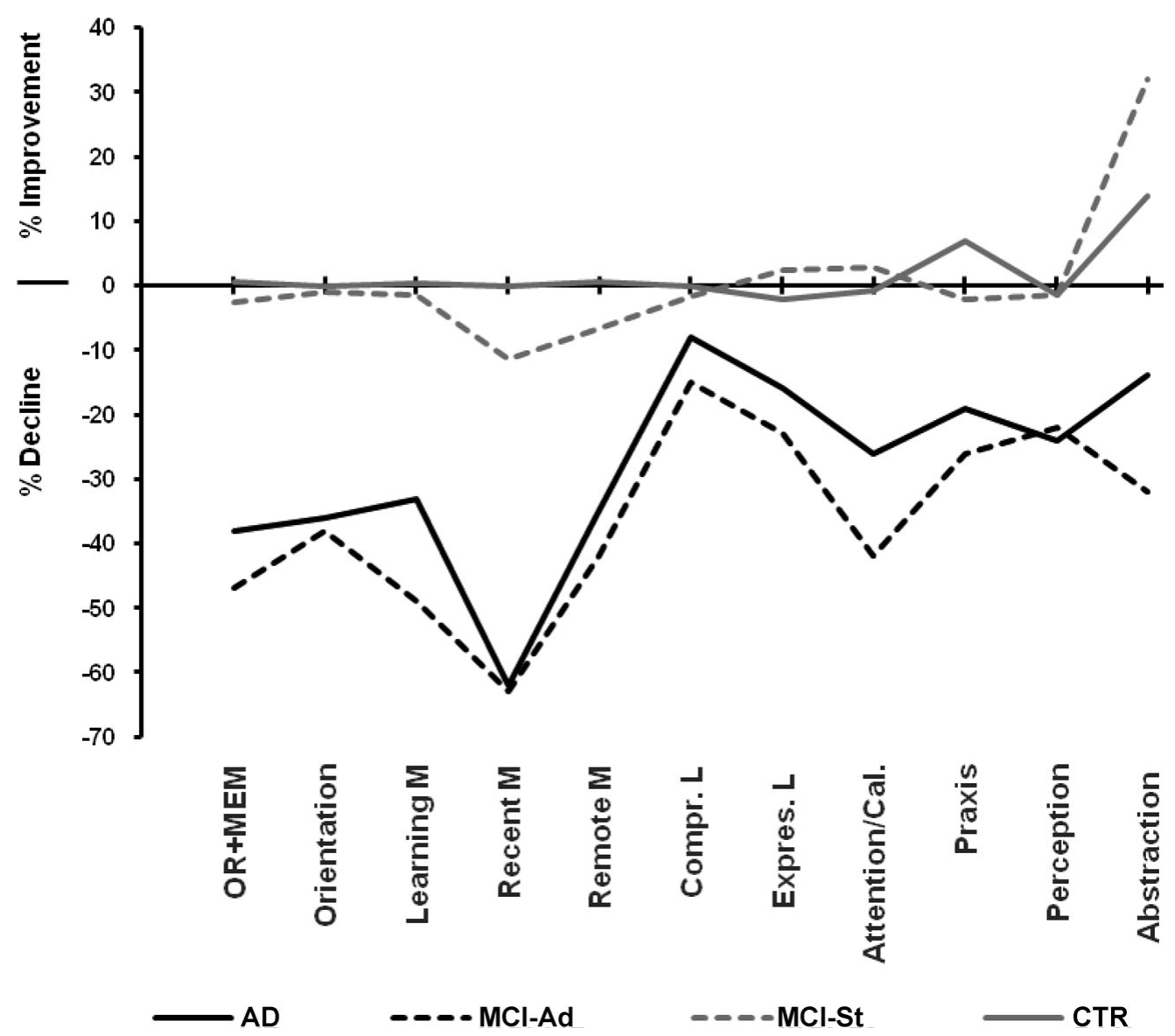

Figure 3. Longitudinal changes after five years: Improvement and decline (\%) on CAMCOG subscales. AD = Alzheimer's disease; $M C I-A d=$ mild cognitive impairment converted to $A D ; M C I-S t=$ mild cognitive impairment-stable; $C T R=$ control group; OR $+M E M=O$ Orientation + Memory (learning and recent); $M=$ Memory; $\mathrm{L}=$ Language; Compr. = comprehension; Expres. = expression; Cal. = calculation.

affected by age, gender, education, or depression. A higher educational level was associated with better performance on Abstraction, Language (expression and comprehension), Attention/Calculation, Praxis, and Memory (remote). Men performed better on Language (comprehension), Memory (remote), Praxis, and Attention/Calculation. Younger age was related to higher scores on Perception. Depression had no effect on any subscale.

The influence of age, education, and gender was greater in the groups without dementia. A higher educational level was associated with better performance on all the subscales except for Memory (learning). Men performed better on Memory (remote), Language (expression), and Attention/Calculation. Younger age was related to higher scores on Perception and Praxis. Depression had a negative effect on the Orientation subscale and on the OR + MEM variable.

\section{Discussion}

In the baseline assessment, the cognitive decline of patients with $\mathrm{AD}$ was already more apparent.
The main difficulty was identifying those patients with MCI who would go on to develop dementia. In this context, the study presents relevant data for the differential diagnosis of patients with MCIAd. Global scores on the MMSE and CAMCOG produced AUC values with null specificity and sensitivity as regards identifying those patients with MCI who were most likely to convert to AD.

Some baseline scores of MCI-Ad patients were similar to those of the groups without dementia, although on some CAMCOG subscales they scored significantly higher (i.e. on Language [expression], Attention/Calculation, and Abstraction with respect to the MCI-ST group, and on Praxis with respect to the CTR group). These higher scores in the MCI-Ad group could, in the baseline assessment, make differential diagnosis difficult. In the ROC analysis, significant differences at baseline were only observed on the combined variable of $\mathrm{OR}+$ MEM (Orientation + Memory [learning and recent]), the AUC value being moderate but significant. The same finding was obtained after five years. These data are conclusive in terms of 
Table 2. Influence of age, education, gender, and depression on CAMCOG subscale scores at baseline. Multivariate linear regression analysis

\begin{tabular}{|c|c|c|c|c|c|c|c|c|}
\hline \multirow[b]{2}{*}{ SUBSCALES } & \multicolumn{4}{|c|}{$\begin{array}{c}\text { DEMENTIA } \\
(\mathrm{AD}+\mathrm{MCI}-\mathrm{AD})(N=168)\end{array}$} & \multicolumn{4}{|c|}{$\begin{array}{c}\text { NON-DEMENTIA } \\
(\mathrm{MCI}-\mathrm{ST}+\mathrm{CTR})(\mathrm{N}=174)\end{array}$} \\
\hline & B (SE) & $\beta$ & $P$ & $95 \% \mathrm{CI}$ & B (SE) & $\beta$ & $P$ & $95 \% \mathrm{CI}$ \\
\hline Memory (learning) & $\begin{array}{l}R^{2}=0.000 \\
\text { NS }\end{array}$ & & & & $\begin{array}{l}R^{2}=0.000 \\
\text { NS }\end{array}$ & & & \\
\hline Memory (recent) & $R^{2}=0.000$ & & & & $R^{2}=0.052$ & & & \\
\hline Schooling & NS & & & & $0.17(0.05)$ & 0.22 & 0.002 & $0.06,0.29$ \\
\hline Orientation & $R^{2}=0.000$ & & & & $R^{2}=0.075$ & & & \\
\hline Depression & NS & & & & $-0.48(0.18)$ & -0.19 & 0.007 & $-0.84,-0.13$ \\
\hline Schooling & NS & & & & $0.19(0.08)$ & 0.17 & 0.018 & $0.03,0.34$ \\
\hline OR + MEM & $R^{2}=0.000$ & & & & $R^{2}=0.075$ & & & \\
\hline Schooling & NS & & & & $0.68(0.23)$ & 0.21 & 0.004 & $0.22,1.14$ \\
\hline Depression & NS & & & & $-1.10(0.52)$ & -0.15 & 0.037 & $-2.14,-0.06$ \\
\hline Perception & $R^{2}=0.028$ & & & & $R^{2}=0.133$ & & & \\
\hline Schooling & NS & & & & $0.31(0.08)$ & 0.25 & $<0.001$ & $0.14,0.49$ \\
\hline Age & $-0.03(0.01)$ & -0.16 & 0.034 & $-0.06,0.00$ & $-0.05(0.01)$ & -0.23 & 0.001 & $-0.09,-0.02$ \\
\hline Abstraction & $R^{2}=0.094$ & & & & $R^{2}=0.087$ & & & \\
\hline Schooling & $0.57(0.14)$ & 0.30 & $<0.001$ & $0.29,0.86$ & $0.59(0.14)$ & 0.29 & $<0.001$ & $0.30,0.88$ \\
\hline Comprehension $\mathbf{L}$ & $R^{2}=0.128$ & & & & $R^{2}=0.120$ & & & \\
\hline Schooling & $0.30(0.07)$ & 0.29 & $<0.001$ & $0.14,0.45$ & $0.37(0.07)$ & 0.34 & $<0.001$ & $0.22,0.52$ \\
\hline Men & $0.49(0.20)$ & 0.17 & 0.017 & $0.08,0.89$ & NS & & & \\
\hline Memory (remote) & $R^{2}=0.131$ & & & & $R^{2}=0.171$ & & & \\
\hline Schooling & $0.28(0.09)$ & 0.24 & 0.002 & $0.11,0.46$ & $0.35(0.09)$ & 0.26 & $<0.001$ & $0.16,0.53$ \\
\hline Men & $0.76(0.23)$ & 0.24 & 0.001 & $0.30,1.23$ & $0.87(0.21)$ & 0.28 & $<0.001$ & $0.44,1.30$ \\
\hline Praxis & $R^{2}=0.153$ & & & & $R^{2}=0.126$ & & & \\
\hline Schooling & $0.46(0.11)$ & 0.30 & $<0.001$ & $0.24,0.69$ & $0.59(0.15)$ & 0.28 & $<0.001$ & $0.30,0.89$ \\
\hline Men & $0.86(0.29)$ & 0.21 & 0.004 & $0.28,1.45$ & NS & & & \\
\hline Age & NS & & & & $-0.07(0.02)$ & -0.18 & 0.011 & $-0.13,-0.01$ \\
\hline Expression L & $R^{2}=0.188$ & & & & $R^{2}=0.191$ & & & \\
\hline Schooling & $0.93(0.15)$ & 0.43 & $<0.001$ & $0.63,1.24$ & $0.79(0.15)$ & 0.36 & $<0.001$ & $0.49,1.10$ \\
\hline Men & NS & & & & $1.01(0.35)$ & 0.19 & 0.005 & $0.31,1.72$ \\
\hline Attention/Calculation & $R^{2}=0.205$ & & & & $R^{2}=0.171$ & & & \\
\hline Men & $1.68(0.37)$ & 0.32 & $<0.001$ & $0.95,2.41$ & $1.70(0.36)$ & 0.32 & $<0.001$ & $0.98,2.41$ \\
\hline Schooling & $0.56(0.14)$ & 0.28 & $<0.001$ & $0.28,0.84$ & $0.45(0.15)$ & 0.20 & 0.004 & $0.14,0.76$ \\
\hline
\end{tabular}

$R^{2}=$ coefficient of determination; $\mathrm{B}=$ unstandardized coefficient; $\mathrm{SE}=$ standard error; $\beta=$ standardized coefficient; $\mathrm{CI}=$ confidence interval for B; NS = not significant.

the predictive capacity of Orientation + Memory (learning and recent) at baseline, whereas scores on the subscales Memory (remote), Language, Abstraction, Attention/Calculation, and Praxis have no predictive value.

The analysis of independent variables showed that age, education, gender and depression had no influence on the baseline cognitive scores of the groups with dementia on the subscales Orientation and Memory (learning and recent) or on the OR + MEM variable. This finding suggests that the low initial score on these subscales is related more to the disease. By contrast, years of schooling and depression did have a greater influence in the groups without dementia and could explain the mild decline observed for scores on these variables.
Our findings differ from previous studies using the CAMCOG when it comes to the predictive capacity of the subscales Perception (LozanoGallego et al., 1999; Marcos et al., 2006; Armas, 2009), Memory (remote) (Nielsen et al., 1999; Schmand et al., 2000), Abstraction (Williams et al., 2003; Gallagher et al., 2010), Attention/Calculation (Lozano-Gallego et al., 1999; Nielsen et al., 1999; Williams et al., 2003), and Language (Nielsen et al., 1999; Oulhaj et al., 2009). Specifically, the present study showed that baseline scores on these subscales were highly influenced by education and, to a lesser extent, by gender and age, which could be the reason for the discrepancies. In the initial assessment of cognitive impairment, these variables would not only have an effect in terms of lower discriminant and predictive capacity but may also 
act as confounders, especially in those MCI patients with a higher educational level and who go on to develop dementia.

As regards longitudinal changes in subscale scores, the data indicate that patients with dementia show a greater decline in Orientation and Memory (learning, recent). The remaining variables either showed a mild decrease (Memory [remote], Attention/Calculation, Language [expression and comprehension], Praxis, and Abstraction) or an intermediate decline (Perception). These differences could be explained using Cattell and Horn's concepts of fluid and crystallized intelligence (Horn, 1982). The former subscales would align more with fluid intelligence (genetic mental ability) and they would tend to decline with older age or disease. Conversely, the latter subscales would be closer to crystallized intelligence (experiential mental ability) and would be significantly influenced by education, showing a stronger tendency toward stability.

The data suggest that in terms of diagnostic discrimination at baseline it would be useful, in the neuropsychological assessment, to complement the global scores on the cognitive assessment scales with information from the Orientation and Memory (learning and recent) subscales, or with specific neuropsychological tests that consider these aspects (Tabert et al., 2006; Dierckx et al., 2009; Chapman et al., 2010; Lekeu et al., 2010; Rami et al., 2010). Moreover, even with the range of data obtained here, the neuropsychological assessment alone is insufficient for a full and accurate diagnosis at baseline. In this regard, the detection of an $\mathrm{AD}$ biomarker would seem to be essential to resolve diagnostic uncertainty in the initial presentation. Medial temporal lobe atrophy, levels of $\mathrm{A} \beta 1-42$ and tau proteins in cerebrospinal fluid, decreased blood flow in hippocampal regions, and reduced metabolism in bilateral temporo-parietal areas are documented as being the most powerful biomarkers (Dubois et al., 2007), albeit without achieving complete accuracy. At all events, the biomarker threshold needs to be clarified so as to differentiate pathological cognitive decline from the normal decline that can occur with aging. At present, therefore, the combination of biological markers and neuropsychological tests would seem to offer the most realistic solution as regards predicting the conversion of MCI to AD.

This study presents a number of limitations. First, there may be a selection bias in relation to patients with dementia, since the inclusion criterion of having neuropsychological assessments over a consecutive five-year period excluded several cases. Second, the educational level of the sample, drawn from a semi-rural context, is low, and scores on some of the subscales could vary in more highly educated populations. Third, although the results for the groups with dementia present a higher degree of certainty, the same cannot be said for the groups without dementia, and it is possible that some of the latter participants might go on to develop dementia. This aspect could be addressed through a longer follow-up, especially in the group with MCI-St. Fourth, although the MCI groups included no cases of psychotic disorder some of the patients had suffered a cerebrovascular accident (CVA; $n=7)$, a factor that could be relevant when comparing the present results with those of studies in which cases of CVA were excluded. Fifth, the number of MCI cases is too small to obtain totally conclusive results. Finally, there could be a bias related to the results in the control group, since data from controls were not collected during the same period as were the data relating to the other three study groups.

\section{Conflict of interest}

None.

\section{Description of authors' roles}

J.L. Conde-Sala was the principal investigator, designed the study, and wrote the paper. J. Garre-Olmo, J. Vilalta-Franch, and S. LópezPousa reviewed the methodological and statistical analysis. J. Llinàs-Reglà, $O$. Turró-Garriga, $M$. Lozano-Gallego, M. Hernández-Ferrándiz, and I. Pericot-Nierga contributed to the recruitment and diagnoses of patients, to the data collection and analysis at the Dementia Unit, Hospital de Santa Caterina, Salt, Spain. In addition, all authors reviewed drafts of the paper prior to publication.

\section{References}

American Psychiatric Association (2000). Manual Diagnóstico y Estadístico de Los Trastornos Mentales, 4th edn, texto revisado (DSM-IV-TR). Barcelona, Spain: Masson.

Armas, J. (2009). Clinical and neuropsychological risk factors to conversion from mild cognitive impairment to Alzheimer. Alzheimer's E Dementia, 5, P382.

Blessed, G., Tomlinson, B. E. and Roth, M. (1968). The association between quantitative measures of dementia and of senile change in the cerebral grey matter of elderly subjects. British fournal of Psychiatry, 114, 797-811.

Cabranes, J. A. et al. (2004). Relevance of functional neuroimaging in the progression of mild cognitive impairment. Neurological Research, 26, 496-501.

Chapman, R. M. et al. (2010). Predicting conversion from mild cognitive impairment to Alzheimer's disease using 
neuropsychological tests and multivariate methods. Fournal of Clinical and Experimental Neuropsychology, 13, 1-13.

Dierckx, E. et al. (2009). Verbal cued recall as a predictor of conversion to Alzheimer's disease in mild cognitive impairment. International fournal of Geriatric Psychiatry, 24, 1094-1100.

Dubois, B. et al. (2007). Research criteria for the diagnosis of Alzheimer's disease: revising the NINCDS-ADRDA criteria. Lancet Neurology, 6, 734-746.

Folstein, M. F., Folstein, S. E. and McHugh, P. R. (1975). "Mini-mental state": a practical method for grading the cognitive state of patients for the clinician. Fournal of Psychiatry Research, 12, 397-408.

Gallagher, D. et al. (2010). Detecting prodromal Alzheimer's disease in mild cognitive impairment: utility of the CAMCOG and other neuropsychological predictors. International fournal of Geriatric Psychiatry, 25, 1280-1287.

Heinik, J. and Shaikewitz, D. (2009). The clock drawing test-modified and integrated approach (CDT-MIA) as an instrument for detecting mild cognitive impairment in a specialized outpatient setting. Fournal of Geriatric Psychiatry and Neurology, 22, 171-180.

Horn, J. L. (1982). The theory of fluid and crystallized intelligence in relation to concepts of cognitive psychology and aging in adulthood. In F. I. M. Craik and S. Trehud (eds.), Aging and Cognitive Processes (pp. 237-278). New York: Plenum.

Lekeu, F. et al. (2010). The California Verbal Learning Test and other standard clinical neuropsychological tests to predict conversion from mild memory impairment to dementia. Fournal of Clinical and Experimental Neuropsychology, 32, 164-173.

López-Pousa, S., Vilalta-Franch, J., Llinàs-Reglà, J., Garre-Olmo, J. and Román, G. C. (2004). Incidence of dementia in a rural community in Spain: the Girona cohort study. Neuroepidemiology, 23, 170-177.

Lozano-Gallego, M., Vilalta-Franch, J., Llinàs-Reglà, J. and López-Pousa, S. (1999). The Cambridge Cognitive Examination as a tool for detection of dementia. Revista de Neurología, 28, 348-352.

Lozano-Gallego, M., Llinàs-Reglà, J., López-Pousa, S. and Vilalta-Franch, J. (2000). El CAMDEX-R en la evaluación clínica de las demencias. Actas Españolas de Psiquiatría, 28, 125-129.

Marcos, A. et al. (2006). Neuropsychological markers of progression from mild cognitive impairment to Alzheimer's disease. American fournal of Alzheimer's Disease and Other Dementias, 21, 189-196.

McKhann, G., Drachman, D., Folstein, M., Katzman, R., Price, D. and Stadlan, E. M. (1984). Clinical diagnosis of Alzheimer disease: report of the NINCDS-ADRDA work group under the auspices of Department of Health and Human Services Task Force on Alzheimer Disease. Neurology, 34, 939-944.

Nielsen, H., Lolk, A., Andersen, K., Andersen, J. and Kragh-Sørensen, P. (1999). Characteristics of elderly who develop Alzheimer's disease during the next two years: a neuropsychological study using CAMCOG. The Odense Study. International fournal of Geriatric Psychiatry, 14, 957-963.

Nunes, P. V. et al. (2008). CAMCOG as a screening tool for diagnosis of mild cognitive impairment and dementia in a Brazilian clinical sample of moderate to high education. International fournal of Geriatric Psychiatry, 23, 1127 1133.

Oulhaj, A., Wilcock, G. K., Smith, A. D. and de Jager, C. A. (2009). Predicting the time of conversion to MCI in the elderly: role of verbal expression and learning. Neurology, 73, 1436-1442.

Peña-Casanova, J. et al. for the NORMACODEM Group (2005). Diagnostic value and test-retest reliability of the Blessed Dementia Rating Scale for Alzheimer's disease: data from the NORMACODEM project. Neurologia, 20, 349-355.

Portet, F. et al. for the MCI Working Group of the European Consortium on Alzheimer's Disease (EADC) (2006). Mild cognitive impairment (MCI) in medical practice: a critical review of the concept and new diagnostic procedure. Report of the MCI Working Group of the European Consortium on Alzheimer's Disease. Fournal of Neurology, Neurosurgery \& Psychiatry, 77, 714-718.

Rami, L., Bosch, B., Sanchez-Valle, R. and Molinuevo, J. L. (2010). The memory alteration test (M@T) discriminates between subjective memory complaints, mild cognitive impairment and Alzheimer's disease. Archives of Gerontology and Geriatrics, 50, 171-174.

Roth, M. et al. (1986). CAMDEX: a standardised instrument for the diagnosis of mental disorder in the elderly with special reference to the early detection of dementia. British Fournal of Psychiatry, 149, 698-709.

Roth, M., Huppert, F. A., Tym, E. and Mountjoy, C. Q. (1998). CAMDEX-R: The Cambridge Examination for Mental Disorders of the Elderly - Revised. Cambridge: Cambridge University Press.

Schmand, B., Walstra, G., Lindeboom, J., Teunisse, S. and Jonker, C. (2000). Early detection of Alzheimer's disease using the Cambridge Cognitive Examination (CAMCOG). Psychological Medicine, 30, 619-627.

Tabert, M. H. et al. (2006). Neuropsychological prediction of conversion to Alzheimer disease in patients with mild cognitive impairment. Archives of General Psychiatry, 63, 916-924.

Vilalta-Franch, J., Llinàs-Reglà, J., Lopez-Pousa, S., Amiel, J. and Vidal, C. (1990). The Cambridge Mental Disorders of the Elderly Examination: validation of the Spanish adaptation. Neurología, 5, 117-120.

Vilalta-Franch, J., López-Pousa, S. and Llinàs-Reglà, J. (2000). The prevalence of dementias in a rural area: a study in Girona. Revista de Neurología, 30, 1026-1032.

Williams, J. G., Huppert, F. A., Matthews, F. E. and Nickson, J. (2003). Performance and normative values of a concise neuropsychological test (CAMCOG) in an elderly population sample. International fournal of Geriatric Psychiatry, 18, 631-644. 